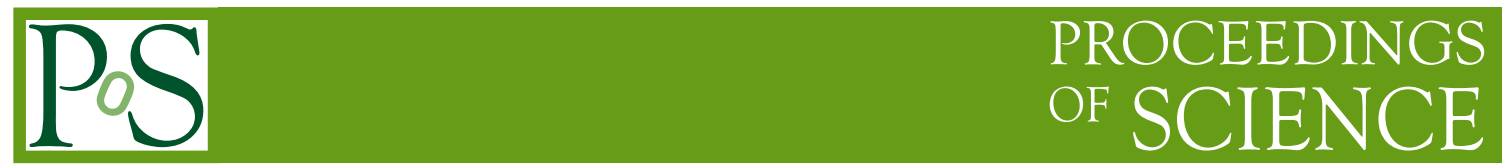

\title{
Evolution of the Universe in the Inert Doublet Model
}

\section{Dorota Sokolowska ${ }^{* \dagger}$}

University of Warsaw

E-mail: dsokefuw.edu.pl

We consider evolution of the Universe after the electroweak symmetry breaking (EWSB) leading to the present inert phase, with a SM-like Higgs boson and scalar dark particles, among them a Dark Matter candidate. We discuss the possibility to have a sequences of the phase transitions instead of a single one leading directly from EW symmeric phase to the inert one.

XXIst International Europhysics Conference on High Energy Physics

21-27 July 2011

Grenoble, Rhône-Alpes, France

\footnotetext{
* Speaker.

${ }^{\dagger}$ I would like to thank I. Ginzburg, K. Kanishev and M. Krawczyk for cooperation and discussions. Work was partly supported by Polish Ministry of Science and Higher Education Grant N N202 230337.
} 


\section{Thermal evolution of the Universe in the IDM}

The Inert Doublet Model (IDM) is a $Z_{2}$-symmetric $2 \mathrm{HDM}$ that may provide the Dark Matter (DM) candidate [1]. There are two scalar $S U(2)$ doublets: a Higgs doublet $\Phi_{S}$ and a dark doublet $\Phi_{D}$. In this model the $Z_{2}$-type symmetry (called the $D$-symmetry) is present: $\Phi_{S} \stackrel{D}{\rightarrow} \Phi_{S}, \Phi_{D} \stackrel{D}{\rightarrow}$ $-\Phi_{D}, S M$ fields $\stackrel{D}{\rightarrow} S M$ fields, leading to the $D$-parity conservation. All components of $\Phi_{D}$ are realized as the massive $D$-odd scalars: two charged $D^{ \pm}$and two neutral $D_{H}$ and $D_{A}$. The lightest particle among them is stable and can be considered as a candidate for the DM particle. This model can be used to describe the evolution of the Universe [2].

The $D$-symmetric potential $V$, which can describe IDM, has the following form:

$V=-\frac{m_{11}^{2}}{2}\left|\Phi_{S}\right|^{2}-\frac{m_{22}^{2}}{2}\left|\Phi_{D}\right|^{2}+\frac{\lambda_{1}}{2}\left|\Phi_{S}\right|^{4}+\frac{\lambda_{2}}{2}\left|\Phi_{D}\right|^{4}+\lambda_{3}\left|\Phi_{S}\right|^{2}\left|\Phi_{D}\right|^{2}+\lambda_{4}\left|\Phi_{S}^{\dagger} \Phi_{D}\right|^{2}+\left(\frac{\lambda_{5}}{2}\left(\Phi_{S}^{\dagger} \Phi_{D}\right)^{2}+h . c\right)$,

with all parameters real and $\lambda_{5}<0$ [2]. Positivity conditions imposed on $V$ guarantee that the extremum with the lowest energy will be the global minimum of the potential (vacuum). We set the Yukawa interaction to Model I of general $2 \mathrm{HDM}$ (only one doublet, $\Phi_{S}$, couples to SM fermions).

We consider thermal evolution of the Lagrangian as in [2], where only the quadratic parameters $m_{i i}^{2}(i=1,2)$ vary with temperature $T$ :

$$
m_{i i}^{2}(T)=m_{i i}^{2}-c_{i} T^{2}(i=1,2),
$$

where $c_{i}=c_{i}\left(\lambda_{1-4} ; g, g^{\prime} ; g_{t}^{2}+g_{b}^{2}\right.$ for $\left.i=1\right), g, g^{\prime}-$ EW gauge couplings, $g_{t}, g_{b}$ - SM Yukawa couplings) [2].

During the evolution the potential $V$ with $m_{i i}^{2}(T)$ may have different ground states [2]. The most general neutral EWSB solution $\left\langle\Phi_{S}\right\rangle^{T}=\frac{1}{\sqrt{2}}\left(0, v_{S}\right),\left\langle\Phi_{D}\right\rangle^{T}=\frac{1}{\sqrt{2}}\left(0, v_{D}\right)$ gives three extrema:

- EW symmetric extremum $(E W s)$ is realized if $v=v_{D}=v_{S}=0$. Here all fermions and bosons are massless and $E W$ symmetry is conserved.

- Inert extremum $\left(I_{1}\right)$ can be realized if $v_{D}=0, v_{S}^{2}=v^{2}=m_{11}^{2} / \lambda_{1}$. In this vacuum state there is the SM-like Higgs particle $h_{S}$ and four dark scalar particles $D_{H}, D_{A}, D^{ \pm}$. We consider IDM with the DM candidate $D_{H}$, where $M_{D^{ \pm}}, M_{D_{A}}>M_{D_{H}}$. Various theoretical and experimental constraints apply for the IDM $[1,2]$. The scalar masses are constrained by EWPT and collider data (LEP II, Tevatron, LHC). The relic density measurements and the direct detection experiments can be used to constrain the DM mass and the DM-Higgs self-coupling $\lambda_{345}$. However, the DM quartic selfcoupling $\lambda_{2}$ cannot be limited this way [2].

- Inertlike extremum $\left(I_{2}\right)$ is mirror-symmetric to $I_{1}$ as $v_{S}=0, v_{D}^{2}=v^{2}=m_{22}^{2} / \lambda_{2}$. There are four scalars $S_{H}, S_{A}, S^{ \pm}$(no DM candidate due to the spontaneous violation of the $D$-symmetry) and the Higgs particle $h_{D}$ with no interaction with the massless fermions (Model I).

- Mixed extremum $(M)$ is a standard 2HDM extremum with $v_{S}, v_{D} \neq 0, v^{2}=v_{S}^{2}+v_{D}^{2}$. Fermions and bosons are massive and there are 5 physical Higgs particles: CP-even $h$ and $H, \mathrm{CP}$-odd $A$ and charged $H^{ \pm}$, none of them can be a DM candidate.

We assume that the Universe today is in the inert phase $I_{1}$ with the DM candidate $D_{H}$. However, various sequences of transitions between different vacua (represented by rays) were possible in the past [2]: 
- $E W s \rightarrow I_{1}$ : rays Ia-c, IIa-b (no $I_{2}, M$ minima during evolution), III ( $I_{2}$ is a local minimum today);

- $E W s \rightarrow I_{2} \rightarrow I_{1}$ : rays IV ( $I_{2}$ is not a local minimum today) and $\mathbf{V}$ ( $I_{2}$ is a local minimum today);

- $E W s \rightarrow I_{2} \rightarrow M \rightarrow I_{1}$ : ray $\mathbf{V I}\left(I_{2}, M\right.$ were global minima in the past $)$.

\section{Numerical study}

For numerical studies we fix the values of the scalar masses that correspond to the medium DM mass region in IDM: $M_{h_{S}}=120 \mathrm{GeV}, M_{D_{H}}=45 \mathrm{GeV}, M_{D_{A}}=115 \mathrm{GeV}, M_{D^{ \pm}}=125 \mathrm{GeV}$, choosing the values of self-couplings $\lambda_{345}, \lambda_{2}$ for each ray [2]. Below we discuss the evolution of mass parameters for three types of sequences (for rays III, V and VI) [2]. In fig.1 mass evolutions of the $W^{ \pm}$boson (proportional to $v(T)$ ) and $t$ quark are shown.

- Ray III: $\lambda_{345}=0.117, \lambda_{2}=0.02, \Omega_{D M} h^{2}=0.0107$ (inside $3 \sigma$ WMAP limit). There is a single 2nd-order phase transition $E W s \rightarrow I_{1}$ (EWSB). Local minimum $I_{2}$ appears at later stages of the evolution of Universe, but it is never a global minimum.

- Ray V: $\lambda_{345}=0.17, \lambda_{2}=0.05, \Omega_{D M} h^{2}=0.0053$ (below $3 \sigma$ WMAP limit). The EWSB is a 2nd-order phase transition, while the final transition $I_{2} \rightarrow I_{1}$ is of the 1st-order. $I_{1}$ and $I_{2}$ coexist for $T<120 \mathrm{GeV}$ and local minimum $I_{2}$ still exists for $T=0$. The discontinuity in $v(T)$ and $m_{t}(T)$ is visible (fig.1b).

- Ray VI: $\lambda_{345}=0.17, \lambda_{2}=0.125, \Omega_{D M} h^{2}=0.0053$ (below $3 \sigma$ WMAP limit). There are three 2nd-order phase transitions $E W s \rightarrow I_{2} \rightarrow M \rightarrow I_{1}$, which lead to the continuous evolution of the physical parameters (fig.1c) and no coexistence of minima for any $T$.

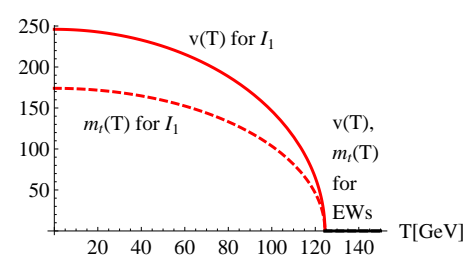

(a) ray III

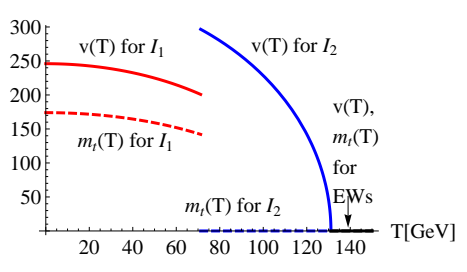

(b) ray $\mathrm{V}$

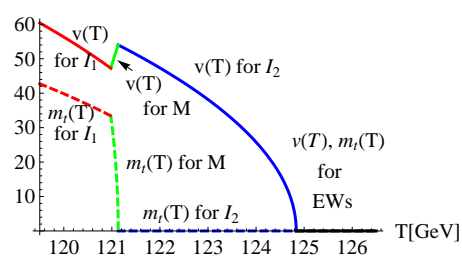

(c) ray VI

Figure 1: Evolution of v.e.v $v(T)$ and top mass $m_{t}(T)$ for different rays [2].

\section{References}

[1] N. G. Deshpande and E. Ma, Phys. Rev. D 18 (1978) 2574; R. Barbieri et al., Phys. Rev. D 74 (2006) 015007; L. Lopez Honorez et al., JCAP 0702 (2007) 028; T. Hambye et al., JHEP 0907 (2009) 090 [Erratum-ibid. 1005 (2010) 066]; E. M. Dolle and S. Su, Phys. Rev. D 80 (2009) 055012; E. Dolle et al., Phys. Rev. D 81, 035003 (2010).

[2] I. F. Ginzburg et al., Phys. Rev. D 82, 123533 (2010); D. Sokołowska, arXiv:1107.1991 [hep-ph], arXiv:1104.3326 [hep-ph]. 